\title{
Cosmic rays in star forming galaxies
}

\section{Martin Pierrick*}

Institut de Recherche en Astrophysique et Planétologie, UPS/CNRS, UMR5277,

31028 Toulouse cedex 4, France

E-mail: pierrick.martineirap.omp.eu

The current generation of high-energy and very-high-energy telescopes made it possible to probe a new class of gamma-ray emitters: star-forming galaxies. A very likely process for the generation of a significant fraction of the observed gamma-rays is the interaction of cosmic rays with the interstellar medium. Thanks to Fermi/LAT observations, a population study was recently performed over a sample of more than 60 luminous infrared galaxies. This revealed an unexpected, almost linear correlation between star formation tracers and $100 \mathrm{MeV}-100 \mathrm{GeV}$ luminosity. I present a model for the non-thermal emissions from steady-state cosmic-ray populations interacting with the interstellar medium in star-forming galaxies, with the goal of establishing whether the current experimental data in the $\mathrm{GeV}$ range can constrain theories on cosmic ray acceleration and transport. I then discuss the extension of such a work to non-thermal emissions in other wavebands.

Cosmic Rays and the InterStellar Medium

24-27 June 2014

Montpellier, France

${ }^{*}$ Speaker. 


\section{Introduction}

Thanks to the current generation of high-energy and very-high-energy telescopes (such as the Fermi/LAT or H.E.S.S., respectively), a new class of gamma-ray sources has emerged: starforming galaxies. The gamma-ray emission from these galaxies is not dominated by accretion and ejection processes onto a central supermassive black hole. Instead, it is connected to the star formation activity in these systems: either nearby objects with a relatively normal, Milky Way-like, star formation activity such as the Andromeda galaxy or the Magellanic Clouds, or more distant objects having a high rate of star formation (or rather a high rate of star formation per unit volume), such as the archetypical starbursts M82 and NGC253. A very likely process for the generation of a significant fraction of the observed gamma-rays is the interaction of cosmic rays (CRs) with the interstellar medium (ISM), the emission arising from a mix of inverse-Compton scattering, Bremsstrahlung, and hadronic interactions. That such objects could be detectable with the current gamma-ray instruments was anticipated by several authors [Völk et al. (1996), Pavlidou \& Fields (2001)].

The class of external star-forming galaxies detected in $\mathrm{GeV}$ gamma-rays with the Fermi/LAT contains: the Small Magellanic Cloud (SMC), the Large Magellanic Cloud (LMC), the Andromeda galaxy (M31), the Sculptor galaxy (NGC253), the Cigar galaxy (M82), and two other spiral galaxies, NGC1068 and NGC4945 [Abdo et al. (2010), Abdo et al. (2010), Abdo et al. (2010), Abdo et al. (2010)]. The latter two belong to the class of Seyfert galaxies and host radio-quiet active galactic nuclei (AGN). There are reasons to believe that the gamma-ray signal from both objects originates primarily from CRs and not from the AGN, but the relative contribution of each process to the total emission cannot be considered as definitely established [Ackermann et al. (2012)]. Complementing these 7 detections ${ }^{1}$, upper limits on the $100 \mathrm{MeV}-100 \mathrm{GeV}$ emission were derived for about 60 infrared-bright galaxies covering more than 3 orders of magnitude in star formation rate. In the TeV domain, the two starbursts M82 and NGC253 were detected, with VERITAS and H.E.S.S., respectively [Acero et al. (2009), Abramowski et al. (2012), Acciari et al. (2009)]. Emission from the LMC was also detected but was resolved into a handful of isolated sources (a pulsar wind nebula, a supernova remnant, and a superbubble; see [Abramowski et al. (2015)]). The most interesting features of these observations are:

1. A hard spectrum over the $\mathrm{GeV}$ to $\mathrm{TeV}$ domain for M82 and NGC253, or at least harder than that of the interstellar emission of the Milky Way (typically 2.2-2.3 for the starbursts versus 2.7 for our Galaxy).

2. A possible, almost linear correlation between star formation rate or its classical tracers (such as infrared or radio continuum emission) and the $100 \mathrm{MeV}-100 \mathrm{GeV}$ luminosity. This was inferred from the few detections achieved and taking into account the many upper limits available.

The interest of this new class of gamma-ray emitters is that external star-forming galaxies provide different astrophysical setups that can be very useful to test our current understanding of the origin

\footnotetext{
${ }^{1}$ At the time of writing, the detection of the luminous infrared galaxy NGC2146 was claimed by [Tang et al. (2014)], bringing the sample of GeV-detected objects to 8. This galaxy will not be discussed in this paper, though.
} 
and life cycle of galactic cosmic rays (those having energies up to $\sim 10^{15} \mathrm{eV}$ ). Changing galaxy size and mass, gas distribution and average density, star formation history, large-scale galactic outflow properties...etc, may result in a galactic cosmic-ray population which differ from that of the Milky Way (for instance in terms of density or spectrum). If our theories on galactic cosmic rays are correct, we should be able to account for these variations from one system to another.

Before and after the launch of the Fermi satellite, the gamma-ray emission from individual starbursts was studied by several authors, with the focus being mainly on M82 and NGC253 [Persic et al. (2008), de Cea del Pozo et al. (2009), Lacki et al. (2011), Paglione \& Abrahams (2012)]. The purpose of the work summarised here is to provide a more global picture of the evolution of the gamma-ray interstellar emission from star-forming galaxies, in the context of the recent population study conducted on the basis of Fermi/LAT observations. I defined a global framework by fixing as many CR-related parameters as possible from Milky Way studies, leaving only a few global quantities as independent variables.

The paper is organised as follows: in a first section, a generic model for the transport of cosmic rays and the associated non-thermal interstellar emissions in star-forming galaxies is briefly introduced; in a second section, a selection of results is presented, focusing on the $100 \mathrm{MeV}-100 \mathrm{GeV}$ emission; in a third and last section, some prospects for extending this work to other wavebands are discussed. For every aspect of the work presented here, more details and references can be obtained from the full study published in [Martin (2014)].

\section{Model of star-forming galaxies}

The model is based on a modified version of the GALPROP public code, which was originally designed for modelling the propagation of CRs and their interactions with the ISM in our Galaxy. Synthetic galaxies are two-dimensional in space with cylindrical symmetry, and are defined by a disk radius $R_{\max }$ and a halo height $z_{\max }$. Then, generic prescriptions are defined for the various interstellar medium components: gas, magnetic field, interstellar radiation field, and CR sources:

- The gas distribution is assumed to be an exponential disk of atomic gas, on top of which come ring or core distributions of molecular gas with different extents and densities. The gas distribution will set many other properties of the ISM, as explained below. For a given galaxy size and gas distribution, the molecular gas density is the independent variable that sets the output non-thermal emission.

- The magnetic field strength depends on the gas surface density to a certain exponent and decreases exponentially with height above the galaxy plane. The exponent of the relation was set in order to match the observed far-infrared to radio continuum correlation. No global or local topology was defined for the field.

- The interstellar radiation field includes three components: cosmic microwave background, stellar, and infrared. The latter two were modelled based on works by [Draine \& Li (2007), Draine et al. (2007)]. The stellar component has a uniform, average mid-plane value. The infrared component is proportional to the star formation rate surface density (defined below). Both have an energy density decreasing exponentially with distance above/below the galactic plane. 
- The distribution of CR sources follows the distribution of star formation rate surface density, which is obtained from the distribution of gas surface density through the Schmidt-Kennicutt relation. The CR input power is proportional to the total star formation rate and its normalisation is obtained from Milky Way studies: $7.10 \times 10^{40} \mathrm{erg} \mathrm{s}^{-1}$ in nuclei and $1.05 \times 10^{39}$ $\mathrm{erg} \mathrm{s}^{-1}$ in electrons for a total star formation rate of $1.9 \mathrm{M}_{\odot} \mathrm{yr}^{-1}$. The injection spectra for CR nuclei and electrons are taken from a typical GALPROP run (run z04LMPDS in [Strong et al. (2010)]).

The transport of CRs was assumed to occur by energy-dependent diffusion in a homogeneous and isotropic way. The form and value of the spatial diffusion coefficient was taken from GALPROP run z04LMPDS. Cosmic-ray acceleration and transport can be expected to proceed differently in environments that differ widely from average Milky Way conditions, but it is interesting enough to assess the effects of global galactic properties for a given CR source and transport scheme. Moreover, the impact of environmental conditions on CR acceleration and transport is poorly constrained at the experimental level.

Based on that model, a total of 88 synthetic galaxies were simulated, with sizes from 4 to 40 $\mathrm{kpc}$, several gas distributions, and spanning about six orders of magnitude in star formation rate.

\section{Main results}

I focus here on how the $100 \mathrm{MeV}-100 \mathrm{GeV}$ luminosity varies with global galactic properties, in the context of the recent population study conducted on the basis of Fermi/LAT observations. The top panel of Fig. 1 shows the relation between total infrared luminosity and gamma-ray luminosity for the full sample of synthetic galaxies, compared to the correlation determined experimentally; the bottom panel shows the ratio of both luminosities as a function of total infrared luminosity and reveals trends that are not easily discernible in the previous, log-log plot.

The predicted level of gamma-ray emission for the 88 simulated galaxies matches the observed level for star formation rates of $\sim 1-10 \mathrm{M}_{\odot} / \mathrm{yr}$. At higher star formation rates, the ratio of luminosities flattens out to a value of about $10^{-4}$ and the predicted luminosities follow the observed trend. At lower star formation rates, the luminosities deviate from the observed trend and fall short of the expected values. This occurs because of a lower cosmic ray to gamma-ray conversion efficiency, which results from smaller confinement volumes and lower average gas densities in the smaller galaxies. This deviation may be partly explained by the fact that only interstellar gammaray emission is modelled here, while total gamma-ray emission is used to determine the observed correlation. In particular, in the case of the Magellanic Clouds, the only detected systems at low star formation rate, a non-negligible contribution from isolated objects such as pulsars or supernova remnants cannot be excluded.

Overall, the observed correlation can be accounted for to a large extent without major modifications to the global scheme inferred for CRs in the Milky Way. In terms of CR transport, the present results suggest that using a single diffusion coefficient and the assumption that CRs experience large-scale volume-averaged interstellar conditions are enough to account for the population constraints available today. There is, however, no universal relation between high-energy gamma-ray luminosity and star formation activity, as illustrated by the scatter introduced by different galactic global properties and the downturn in gamma-ray emission at the low end. 


\section{Extension to other wavebands}

The current population of $\mathrm{GeV}$-detected star-forming galaxies does not seem to bring strong constraints on CRs, likely because the sample is still too limited and uncertainties are still too large. Yet, a more global study can (and should) be performed by considering non-thermal emission in other wavebands, namely soft gamma-rays $\sim 100 \mathrm{keV}-10 \mathrm{MeV}$, very high energy gamma-rays $\sim 100 \mathrm{GeV}-100 \mathrm{TeV}$, and radio continuum $\sim 100 \mathrm{MHz}-10 \mathrm{GHz}$.

In soft gamma-rays, the interstellar emission arises predominantly from inverse-Compton scattering, with a non-negligible contribution from secondary leptons in the densest galaxies. This signal, however, may be dominated by other sources such as compact objects or positron annihilation up to at least $511 \mathrm{keV}$ [Bouchet et al. (2011)]. An attempt to extract the soft gamma-ray interstellar signal from a starburst was recently made on NGC253 with NuSTAR, and resulted in upper limits [Wik et al. (2014)]. Beyond $511 \mathrm{keV}$, the difficulty may be instrumental: current instruments such as INTEGRAL/SPI are not sensitive enough, and no successor is currently planned.

In TeV gamma-rays, two starbursts were detected and the sample might be somewhat enriched once the Cherenkov Telescope Array is operational. Interestingly, the model presented above seems to predict softer spectra than observed for NGC253 and M82 in the TeV range, implying that combining the $\mathrm{GeV}$ and $\mathrm{TeV}$ observations in the framework of a global model may bring some constraints on galactic CRs, especially on CR transport and the role of galactic winds. This will be addressed in a forthcoming publication.

In radio continuum, prospects are very good because of a large sample of detected objects. Nearly 2000 star-forming galaxies were considered in past studies of the well-known far infrared - radio continuum correlation [Yun et al. (2001)]. Observations in these bands can provide a high level of spatial and spectral details, and the sensitivities are such that the phenomenon can be probed up to redshift $z \sim 2$ and beyond. In addition, upcoming instruments such as SKA will very likely allow a major step forward. The model presented above can readily predict radio synchrotron luminosities and spectral indices at various frequencies. The results currently available indicate a downturn of the far infrared - radio continuum correlation at low star formation rates, similar to that observed in the $100 \mathrm{MeV}-100 \mathrm{GeV}$ luminosity. The predicted spectral indices at $1.4 \mathrm{GHz}$ of most synthetic galaxies are in the range $-0.7--0.9$, in agreement with observations [Niklas et al. (1997)]. Yet, a complete analysis covering a broad radio spectral range and including other sources of continuum emission such as thermal Bremsstrahlung remains to be done.

\section{References}

[Abdo et al. (2010)] Abdo, A. A., Ackermann, M., Ajello, M., et al. 2010, A\&A, 523, L2+

[Abdo et al. (2010)] Abdo, A. A., Ackermann, M., Ajello, M., et al. 2010, A\&A, 512, A7+

[Abdo et al. (2010)] Abdo, A. A., Ackermann, M., Ajello, M., Atwood, W. B., \& Fermi LAT Collaboration. 2010, ApJ, 709, L152

[Abdo et al. (2010)] Abdo, A. A., Ackermann, M., Ajello, M., et al. 2010, A\&A, 523, A46+

[Abramowski et al. (2012)] Abramowski, A., Acero, F., Aharonian, F., et al. 2012, ApJ, 757, 158

[Abramowski et al. (2015)] Abramowski, A., Aharonian, F., Ait Benkhali, F., et al. 2015, Science, 347, 406 
[Acciari et al. (2009)] Acciari, V. A., Aliu, E., Arlen, T., et al. 2009, Nature, 462, 770

[Acero et al. (2009)] Acero, F., Aharonian, F., Akhperjanian, A. G., et al. 2009, Science, 326, 1080

[Ackermann et al. (2012)] Ackermann, M., Ajello, M., Allafort, A., et al. 2012, ApJ, 755, 164

[Bouchet et al. (2011)] Bouchet, L., Strong, A. W., Porter, T. A., et al. 2011, ApJ, 739, 29

[de Cea del Pozo et al. (2009)] de Cea del Pozo, E., Torres, D. F., \& Rodriguez Marrero, A. Y. 2009, ApJ, 698,1054

[Draine et al. (2007)] Draine, B. T., Dale, D. A., Bendo, G., et al. 2007, ApJ, 663, 866

[Draine \& Li (2007)] Draine, B. T. \& Li, A. 2007, ApJ, 657, 810

[HESS collaboration (2014)] Komin, N. \& HESS collaboration, 2014, 5th Fermi Symposium

[Lacki et al. (2011)] Lacki, B. C., Thompson, T. A., Quataert, E., Loeb, A., \& Waxman, E. 2011, ApJ, 734, 107

[Martin (2014)] Martin, P. 2014, A\&A, 564, A61

[Niklas et al. (1997)] Niklas, S., Klein, U., \& Wielebinski, R. 1997, A\&A, 322, 19

[Paglione \& Abrahams (2012)] Paglione, T. A. D. \& Abrahams, R. D. 2012, ApJ, 755, 106

[Pavlidou \& Fields (2001)] Pavlidou, V. \& Fields, B. D. 2001, ApJ, 558, 63

[Persic et al. (2008)] Persic, M., Rephaeli, Y., \& Arieli, Y. 2008, A\&A, 486, 143

[Strong et al. (2010)] Strong, A. W., Porter, T. A., Digel, S. W., et al. 2010, ApJ, 722, L58

[Tang et al. (2014)] Tang, Q.-W., Wang, X.-Y., \& Tam, P.-H. T. 2014, ApJ, 794, 26

[Völk et al. (1996)] Völk, H. J., Aharonian, F. A., \& Breitschwerdt, D. 1996, Space Sci. Rev., 75, 279

[Wik et al. (2014)] Wik, D. R., Lehmer, B. D., Hornschemeier, A. E., et al. 2014, ArXiv e-prints

[Yun et al. (2001)] Yun, M. S., Reddy, N. A., \& Condon, J. J. 2001, ApJ, 554, 803 

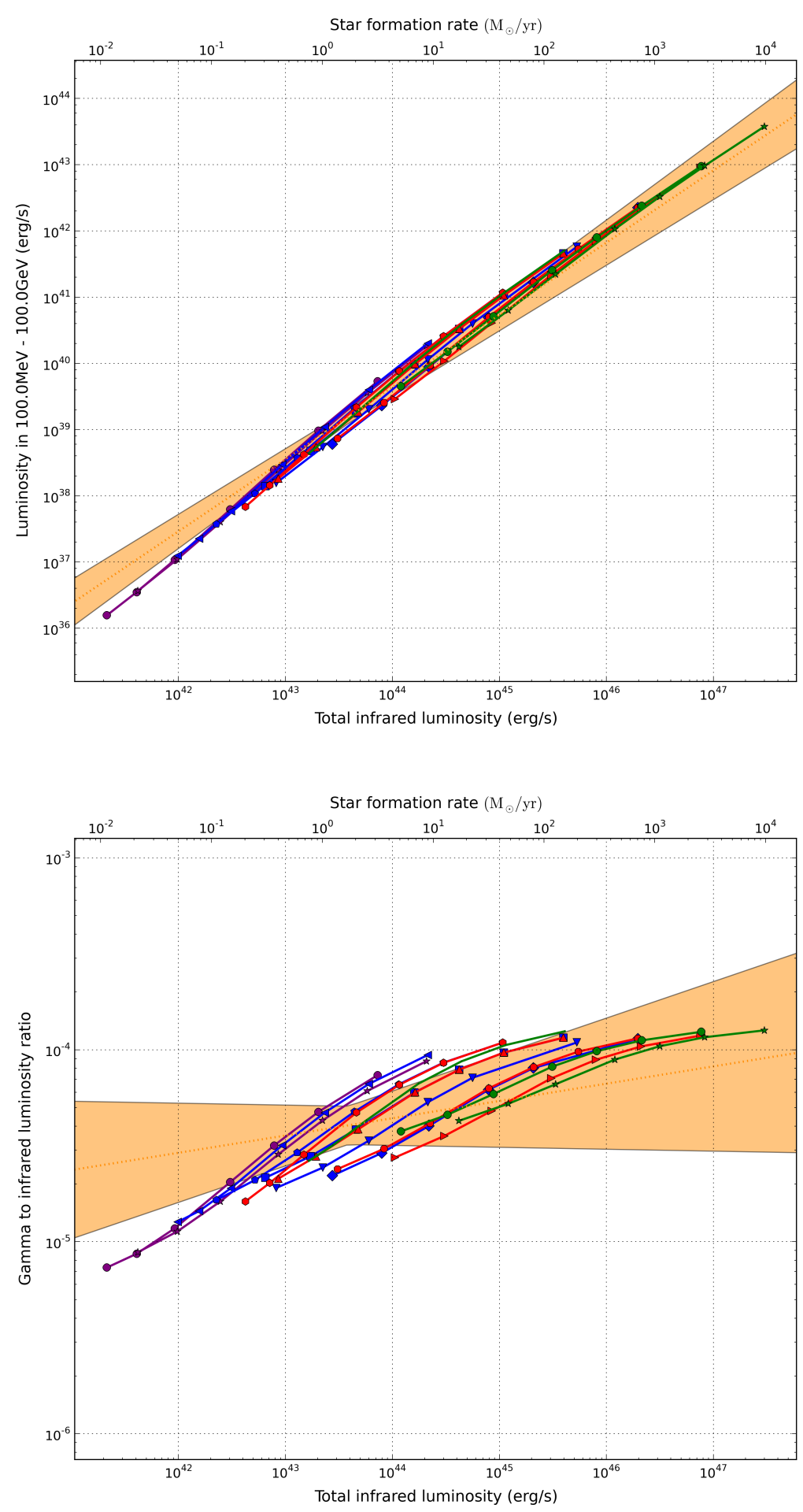

Figure 1: Top: Luminosity in the $100 \mathrm{MeV}-100 \mathrm{GeV}$ band as a function of total infrared luminosity and star formation rate for the complete sample of models. The orange region is the uncertainty range of the correlation determined experimentally. Bottom: Ratio of $100 \mathrm{MeV}-100 \mathrm{GeV}$ to total infrared luminosity. 